\title{
A Demonstrator of the GINSENG-Approach to Performance and Closed Loop Control in WSNs
}

\author{
F. Büsching, W.-B. Pöttner, D. Brökelmann, G. von Zengen, R. Hartung, K. Hinz and L. Wolf \\ Technische Universität Braunschweig \\ Institute of Operating Systems and Computer Networks (IBR) \\ Mühlenpfordtstr. 23, 38106 Braunschweig \\ Email: [buesching | poettner I broeke I vonzeng | hartung I hinz I wolf] @ibr.cs.tu-bs.de
}

\begin{abstract}
The overall goal of GINSENG is a wireless sensor network that will meet application-specific performance targets, and that will be proven in a real industry setting where performance is critical.

This demonstrator shows some of the key outcomes of the GINSENG project such as energy-efficient wireless real-time closed loop control, performance debugging, integration with ERP systems and usability on heterogeneous hardware. Furthermore, it allows the user to interact with the system.
\end{abstract}

\section{INTRODUCTION}

The overall goal of GINSENG [1] is a wireless sensor network that will meet application-specific targets, and that will be proven in a real industry setting where performance is critical. The network has to meet application-specific time and delivery rate targets while the use of WSN technology should result in significant savings in deployment and maintenance costs and offer easy reconfiguration and rapid deployment in adapting to changing business needs or dynamic topology.

Within 3.5 years of research, a significant number of novel ideas have been developed and published. Among those are mechanisms for predictable access to the wireless medium (GinMAC [2]), dynamic topology organization, in-network performance debugging, online reception quality feedback [3] and a distributed middleware system.

To evaluate GINSENG in a real industrial facility, a realworld testbed within a live oil refinery in Portugal has been installed [4] and two separate wireless networks have been used for various experiments. To showcase the GINSENG system, a demonstrator has been created, combining the following three goals. On the one hand, we want to visualize the industrial context GINSENG is designed for. Furthermore, we want to demonstrate some of the key functionality of GINSENG including energy-efficient wireless real-time wireless monitoring and control, integration with backend systems and inoperability between different hardware platforms. Finally, we want to give people the opportunity to interact with the system to make it easier to comprehend.

\section{Demonstrator Hardware Setup}

To emphasize the intended industrial setting, the demonstrator consists of several interconnected tanks, tubes, valves and pumps, all connected wirelessly by a wireless sensor network and powered by batteries. Whereas in the original (refinery)

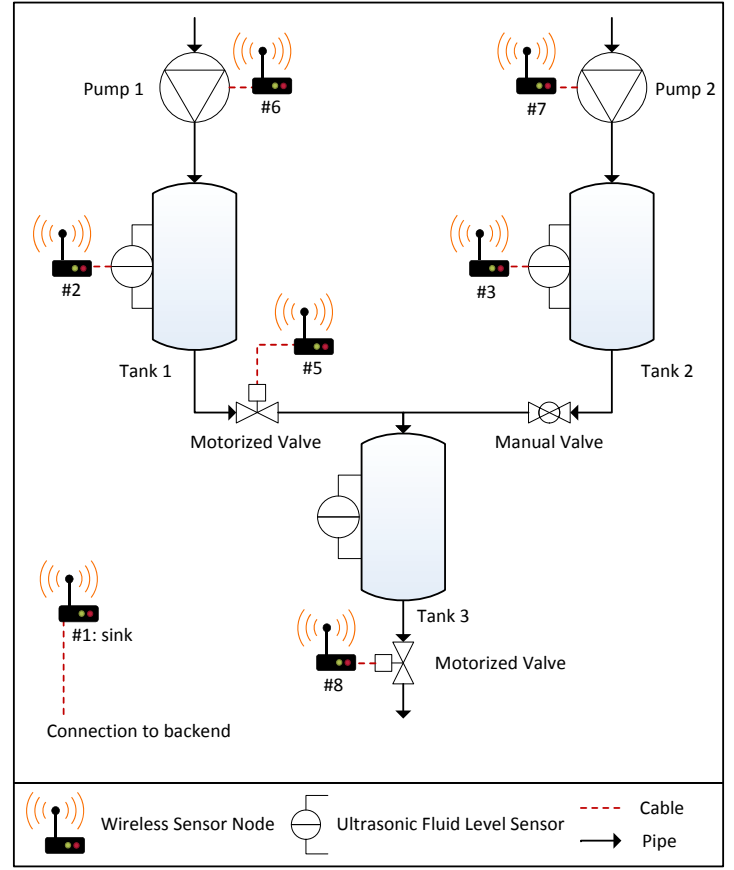

Figure 1. Demonstrator Hardware Setup

setup these components are distributed within the water treatment facility of the refinery, the demonstrator combines them on a vertical plate $\left(2,5 \mathrm{~m}^{2}\right)$ of acrylic glass.

To show GINSENG's industrial context, we have replicated a very simplified version of an industrial process. Figure 1 shows the symbolic hardware setup that we have used and where we implemented some exemplary control loops.

We have used several Wireless Sensor Nodes in this setup, which all are connected to the different sensors and actuators. In addition, we have a mote acting as gateway (sink) that is on the one hand coordinating the wireless network and also communicating with backend systems on the PC via USB. In detail, the interfaces between sensors, actuators and nodes are as follows:

- Fluid Level Sensor: Ultrasonic sensors mounted in the top caps of the tanks constantly measure the distance to the liquid's surface. The sensor data is transferred to attached nodes via $\mathrm{I}^{2} \mathrm{C}$.

Published by the IEEE Computer Society 


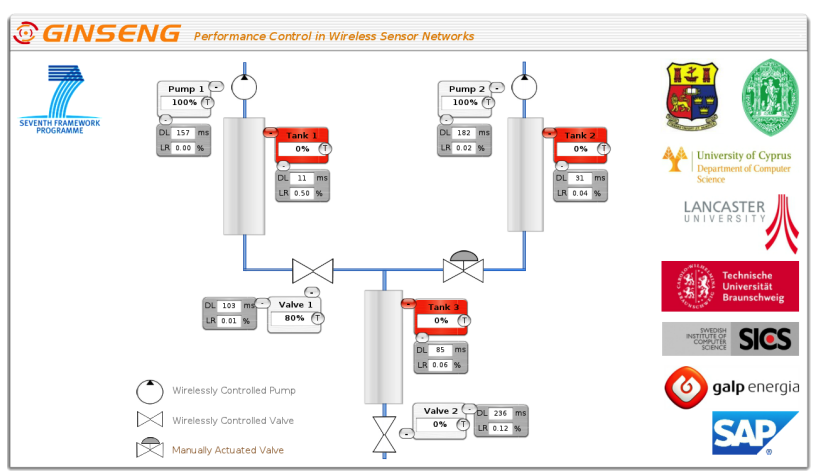

Figure 2. Industrial Process in the GINSENG middleware UI.

- Motorized Valve: Home Heating Valves using the OpenHR20 $0^{1}$ firmware receive control commands from nodes via RS-232.

- Pump: Electric pumps are controlled by nodes via an $\mathrm{I} / \mathrm{O}$ pin using a MOSFET.

\section{Demonstrator Software Setup}

To show what the GINSENG system is capable of, the demonstrator makes use of various functionality as outlined in the following.

\section{A. Wireless Closed Loop Control}

GINSENG is capable of wireless closed loop control. Control loops can either be implemented in the gateway node in case that all sensors and actuators are part of one network tree. Furthermore, control loops can also be closed in the backend in case the loops incorporate components from multiple network trees. However, while the former can ensure real-time behavior, the latter should only be used for non-critical processes. Nevertheless, both variants can be configured using the same backend interface, which then either starts the appropriate functionality in the backend or configures the necessary functionality on the gateway node.

Several wireless control loops are implemented in the demonstrator, whereas all of them are closed on the gateway node. The network keeps the liquid levels in the upper two tanks between 20 and $80 \%$ while tank 3 is kept between 40 and $60 \%$. To dynamically influence the system a user can interact by adjusting a manual valve; the system will react accordingly.

\section{B. Performance Debugging}

The GINSENG system allows operators to closely monitor the networks operation and helps localizing the source of certain problems. While a large number of different metrics are collected and stored in the backend, the two most important metrics for a wireless real-time network are the end-to-end packet loss as well as the end-to-end latency.

\section{Middleware Integration}

One of GINSENG's key features is integration with existing Enterprise Resource Planning (ERP) systems. To show such

\footnotetext{
${ }^{1}$ http://openhr20.sourceforge.net/
}

integration the sink node is connected to the OSGi-based GINSENG middleware [5] running on a standard PC. This demonstrator uses a GUI resembling the industrial process on top of the middleware which shows the current state of all sensors and actuators (see Figure 2) and also includes packet loss and delay.

\section{Hardware heterogeniety}

While the GINSENG project has used a homogeneous testbed of TelosB [6] nodes to simplify things, this demonstrators uses two different hardware platforms. The gateway node is the well-known TelosB node and coordinates the network. Furthermore, all nodes "in the field" are INGA [7] nodes based on different MCU and radio hardware. While the TelosB uses the MSP430 MCU and CC2420 radio, INGA is using at Atmel ATmega and an AT86RF231 radio. Nevertheless, both platforms are supported by Contiki OS and the GINSENG Medium Access Control GinMAC.

\section{SUMMARY}

Within GINSENG it has been shown that deterministic wireless real-time automation in an industrial setting is feasible. In the real-world testbed in a live oil refinery it was shown that the GINSENG system works as reliable as the existing wired system. The presented demonstrator illustrates the functionality of major parts of the system.

\section{ACKNOWLEDGMENT}

This work has been partially supported by the European Commission under the FP7 contract FP7-ICT-224282 (GINSENG).

\section{REFERENCES}

[1] T. O'Donovan, J. Brown, U. Roedig, C. Sreenan, J. do O, A. Dunkels, A. Klein, J. Sa Silva, V. Vassiliou, and L. Wolf, "GINSENG: Performance Control in Wireless Sensor Networks," in 7th Annual IEEE Communications Society Conference on Sensor Mesh and Ad Hoc Communications and Networks (SECON), june 2010, pp. $1-3$.

[2] P. Suriyachai, J. Brown, and U. Roedig, "Time-Critical Data Delivery in Wireless Sensor Networks," in Distributed Computing in Sensor Systems, ser. Lecture Notes in Computer Science, R. Rajaraman, T. Moscibroda, A. Dunkels, and A. Scaglione, Eds. Springer Berlin / Heidelberg, 2010, vol. 6131, pp. 216-229. [Online]. Available: http://dx.doi.org/10.1007/978-3-642-13651-1_16

[3] W.-B. Pöttner, S. Schildt, D. Meyer, and L. Wolf, "Piggy-Backing Link Quality Measurements to IEEE 802.15.4 Acknowledgements," in Mobile Adhoc and Sensor Systems (MASS), 2011 IEEE 8th International Conference on, oct. 2011, pp. $807-812$.

[4] W.-B. Pöttner, L. Wolf, J. Cecilio, P. Furtado, R. Silva, J. Silva, A. Santos, P. Gil, A. Cardoso, Z. Zinonos, J. do O, B. McCarthy, J. Brown, U. Roedig, T. O'Donovan, C. Sreenan, Z. He, T. Voigt, and A. Jugel, "WSN evaluation in industrial environments first results and lessons learned," in Distributed Computing in Sensor Systems and Workshops (DCOSS), 2011 International Conference on, june 2011, pp. 1 -8.

[5] A. Klein and Z. Jerzak, "The GINSENG Middleware for Performance Control in Sensor Networks," in 5th Conference on Management and Control of Production Logistics (MCPL), 2010.

[6] J. Polastre, R. Szewczyk, and D. Culler, "Telos: enabling ultra-low power wireless research," in IPSN '05: Proceedings of the 4th international symposium on Information processing in sensor networks. Piscataway, NJ, USA: IEEE Press, 2005, p. 48.

[7] F. Büsching, U. Kulau, and L. Wolf, "Demo: INGA - An Inexpensive Node for General Applications," in Proceedings of the 9th ACM Conference on Embedded Networked Sensor Systems, ser. SenSys '11. Seattle, WA, USA: ACM, 2011. 